\title{
EL CONTROL EX POST DE OPERACIONES DE CONCENTRACIÓN. ANÁLISIS LEGAL, VENTAJAS Y CONDICIONES PARA SU IMPLEMENTACIÓN
}

\author{
Dario Morales Herrera* \\ Instituto de Competencia y Regulación \\ dario.morales@unmsm.edu.pe \\ Fernando Ballon Estacio** \\ Instituto de Competencia y Regulación \\ fernando.ballon@unmsm.edu.pe
}

Resumen: El control de operaciones de concentración por parte de las autoridades de competencia es una herramienta ampliamente aceptada en el Derecho de la Competencia. Al respecto, los autores analizan la posibilidad de que, bajo el Decreto Legislativo 1034, se puedan perseguir este tipo de conductas. Asimismo, exponen algunas consideraciones con relación a la implementación del control de oficio en la norma de control de operaciones económicas.

Palabras clave: Abuso de posición de dominio, control de concentraciones, Derecho de la Competencia.

\section{EX POST CONTROL OF CONCENTRATION OPERATIONS. LEGAL ANALYSIS, ADVANTAGES AND CONDITIONS FOR ITS IMPLEMENTATION}

\begin{abstract}
Control of concentration operations by competition authorities is a widely accepted tool in Competition Law. In this regard, the authors analyze the possibility that, under Legislative Decree 1034, this type of conduct may be prosecuted. Likewise, they

\footnotetext{
${ }^{*}$ Miembro fundador del Instituto de Competencia y Regulación - ICR. Es egresado de la Maestría en Derecho de la Empresa de la Universidad Nacional Mayor de San Marcos. Actualmente cursa la Maestría en Filosofía con mención en Ética y Políticas Públicas de la Universidad Antonio Ruiz de Montoya. Asociado en Diez Canseco Abogados.

** Miembro fundador del Instituto de Competencia y Regulación - ICR. Asesor Legal en Estudio Muñiz y Oficial de Cumplimiento. Diplomado en Derecho y Política de la Competencia por la Universidad de Chile y egresado de la Maestría en Derecho de la Empresa de la Universidad Nacional Mayor de San Marcos.
} 
present some considerations in relation to the implementation of ex officio control in the regulation of control of economic operations.

Keywords: Abuse of Dominance, Prior Control, Competition Law.

\section{Introducción}

Si bien existen desafíos relacionados con el diseño institucional de la autoridad nacional de competencia, es decir, el Instituto Nacional de Defensa de la Competencia y de la Propiedad Intelectual (en adelante, Indecopi), en cuanto a su independencia, imparcialidad, cumplimiento de plazos, entre otros (Organización para la Cooperación y el Desarrollo Económicos, 2018), el Derecho y Política de Libre Competencia en el Perú ha tenido un desarrollo destacable en los últimos años. Esto fue posible a través de la aprobación de lineamientos, modificaciones legislativas y la reciente promulgación en el año 2019 del Decreto de Urgencia 013-2019, Decreto de Urgencia que establece el control previo de operaciones de concentración empresarial (en adelante, DU 13-2019), que ha de entrar en vigencia en marzo del año 2021.

El DU 13-2019 establece la obligación de solicitar autorización para la realización de aquellas operaciones de concentración empresarial que superen determinados umbrales medidos en función al valor de los ingresos o ventas brutas de las empresas que deseen concentrarse, pues se asume que estas podrían causar una restricción significativa a la competencia en los mercados que correspondan. Sin embargo, ¿qué ocurriría con aquellas operaciones que, a pesar de no superar el umbral, pueden causar un impacto relevante en los niveles de concentración?

En este artículo se analizará la política de control ex post de operaciones de concentración empresarial como herramienta con la que cuentan autoridades de competencia en distintas partes del mundo para hacer frente a este problema y las ventajas y desventajas de su aplicación (apartado 3). En esa línea, evaluaremos si en la legislación peruana se ha previsto esta herramienta a partir de los alcances de la Ley de Represión de Conductas Anticompetitivas (en adelante, la LRCA) y su desarrollo jurisprudencial (apartado 4). Con ello, desarrollaremos algunas de las condiciones que se hacen necesarias para que esta política pueda funcionar de manera óptima (apartado 5).

Para abordar estas cuestiones, es necesario partir de un marco teórico y conceptual que nos permita entender cómo el DU 13-2019 y su enfoque preventivo complementan el sistema represivo y correctivo que representa la LRCA. Como veremos en el apartado 2, 
ambos persiguen los mismos fines (promover la eficiencia económica), pero a través de herramientas diferentes.

\section{Eficiencia y operaciones de concentración empresarial}

Existe una identidad teleológica entre la LRCA y el DU 13-2019, ya que ambos establecen en su primer artículo que su finalidad es promover la eficiencia económica para el bienestar de los consumidores ${ }^{1}$. Por el lado de la LRCA, mediante la sanción y corrección ex post de las conductas anticompetitivas, mientras que por el lado del DU 13-2019, a través del control preventivo de las operaciones de concentración empresarial.

La eficiencia económica hace referencia a aquel nivel de producción y precios que, en un mercado determinado, maximiza los beneficios (o excedentes) obtenidos por los productores y los consumidores, dadas unas determinadas condiciones de oferta y demanda. Dicho punto máximo se logra cuando el mercado corresponde a una estructura de competencia en el que el precio es igual al costo marginal de producción. Este es el concepto de eficiencia asignativa, que se alcanza "cuando los consumidores pueden obtener las cantidades de bienes que requieren al precio que están dispuestos a pagar cubriendo el costo de producción" (pp. 39-40), el cual se complementa con los conceptos de eficiencia productiva y eficiencia dinámica (Quintana Sánchez, 2011).

Al respecto, el modelo de duopolio de Cournot puede ser útil para entender la complementariedad entre las políticas de control de conductas y control de concentraciones y sus repercusiones en los niveles de eficiencia en los mercados.

\section{LRCA}

Artículo 1.- Finalidad de la presente

Ley La presente Ley prohíbe y sanciona las conductas anticompetitivas con la finalidad de promover la eficiencia económica en los mercados para el bienestar de los consumidores.

DU 13-2019

Artículo 1.- Objeto

El presente decreto de urgencia tiene por objeto establecer un régimen de control previo de operaciones de concentración empresarial con la finalidad de promover la eficiencia económica en los mercados para el bienestar de los consumidores. 
Figura 1

Modelo de duopolio de Cournot

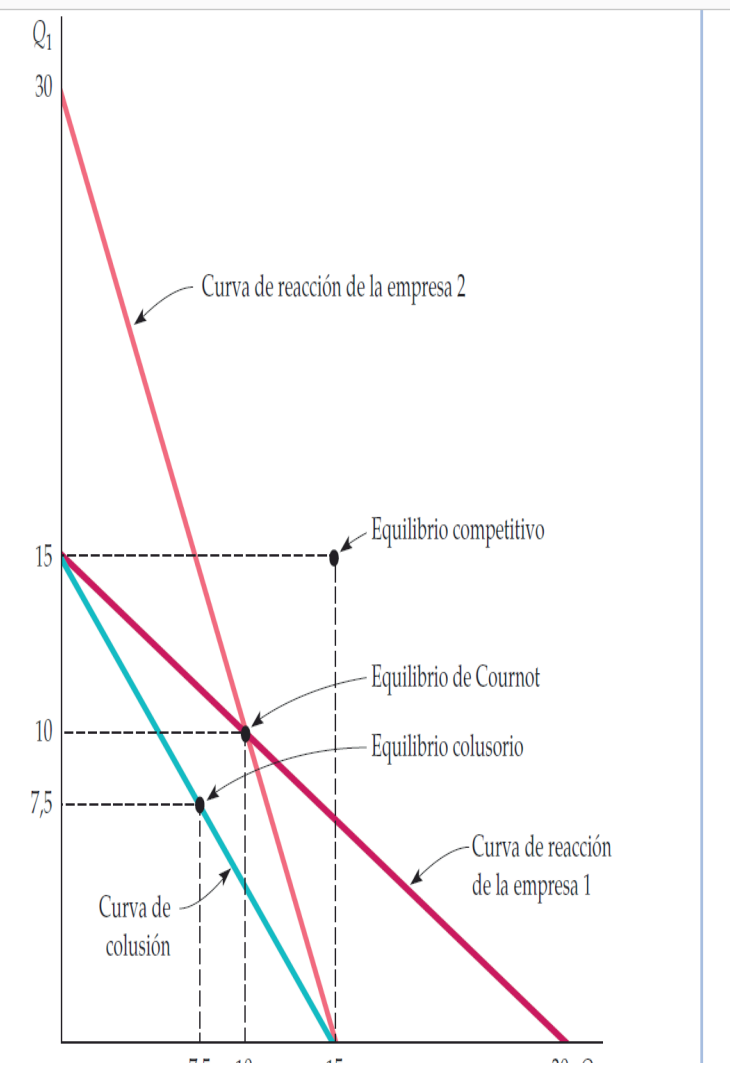

De Microeconomía (p. 520), por Pindyck \& Rubinfeld, 2009. Pearson.

En un mercado de estructura oligopólica, las empresas son interdependientes entre sí, lo que las lleva a adoptar una estrategia comercial en función a la estrategia que adoptan sus competidores. En un duopolio de Cournot se describe el comportamiento de las dos únicas empresas en un mercado hipotético que compiten entre sí en función a las cantidades de producción que fijan, de modo que la decisión de cada una de estas dependerá de la cantidad producida por su competidora hasta el punto en el que logre maximizar su utilidad. Esto es, cuando su ingreso marginal iguala su costo marginal.

Como puede verse en el gráfico, en mercados con esta estructura, las empresas alcanzarán un equilibrio en el que ambas puedan fijar aquella cantidad que permita maximizar sus utilidades considerando la estrategia de su competidora y en función a sus curvas de reacción, que indican la cantidad óptima a fijar ante cualquier posible cantidad determinada por su competidor. No obstante, si las empresas cooperasen, podrían alcanzar una mayor utilidad, reduciendo conjuntamente sus cantidades producidas. Así, tanto en el equilibrio no cooperativo de Cournot como en un escenario de colusión producto de la 
cooperación, las cantidades son mucho menores que aquellas que corresponderían a un equilibrio competitivo en el que el mercado alcanza su máximo nivel de eficiencia.

Una política de control de conductas tiene como finalidad promover la eficiencia, en la medida que conduce del equilibrio de colusión al equilibrio de Cournot, mientras que una política de control preventivo de estructuras evita que el equilibrio del mercado se distancie del equilibrio competitivo o nivel máximo de eficiencia.

Las conductas anticompetitivas en general, incluyendo los actos de concentración, pueden tener por efecto reducir el nivel de eficiencia de un mercado, por lo que, al menos si nos limitamos al análisis teleológico de la LRCA, no habría impedimento para que estos comportamientos también puedan ser revisados ex post por la autoridad.

Sin perjuicio de lo señalado, debe tenerse en cuenta que el análisis puramente estructural no es suficiente para determinar si una operación de concentración es perjudicial, si bien las condiciones estructurales juegan un rol importante. Como reseña Kwoka (2015), la evolución del entendimiento sobre el impacto de las operaciones de concentración se ha reflejado en los cambios a los Lineamientos de Concentraciones Horizontales de los Estados Unidos, partiendo de un enfoque de corte estructuralista en 1968. Luego se reflejó dicho enfoque con herramientas de medición de la variación en los niveles de concentración (como por ejemplo con el IHH) y tests como el del monopolista hipotético en 1982. Pasó después a prever argumentos de eficiencia planteados por las partes en 1984 y a establecer condiciones para aceptarlos en 1997, hasta establecer una teoría más completa del daño en 1992, y una mayor preocupación por la disponibilidad y uso de la evidencia y herramientas más sofisticadas de medición de impacto como el Upward Pricing Pressure en 2010.

Con todo, no pueden condenarse anticipadamente las operaciones de concentración y menos cuando la evidencia es escasa, requiriendo de un balance detenido entre las ganancias de eficiencia y las restricciones a la competencia que pueden producir. En efecto, "Mergers, unlike Price-fixing cartels, involve the integration of combined operations. This difference explains the fact that price fixing is a per se offense while mergers are considered under the rule of reason" (Viscusi, Vernon \& Harrington Jr., 2000, p. 203).

Por esa razón, es importante que la legislación o la jurisprudencia prevean un estándar de prueba,

...since establishing the balance of anti-competitive and pro-competitive effects involves a prediction of the impact of a merger on future market conditions, agencies need to rely on a decisional threshold, i.e., a tipping point beyond which 
sufficient harm to competition is reasonably expected, and which distinguishes mergers that should be prohibited (absent remedies) from those that should be allowed. This is the standard of proof for agency decisions. (OECD, 2016, p. 5)

Sobre estas premisas es que se han diseñado los sistemas de análisis de las operaciones de concentración, que tradicionalmente han tenido un carácter preventivo o ex ante, como el establecido por el DU 13-2019. Dicha norma regula un procedimiento con, entre otras, las siguientes características:

- Umbrales de notificación obligatoria: se fijan umbrales de ventas o ingresos brutos conjuntos de 118000 UIT y un umbral individual de ventas o ingresos brutos de 18000 UIT. En ese sentido, solo los actos de concentración que superen esa valla deben ser notificados al Indecopi para su aprobación (artículo 6).

- "Vía rápida": en los primeros 30 días desde iniciado el procedimiento se determinará si la operación genera preocupaciones por sus impactos en la competencia, con base en la información disponible. De no ser así, la operación se aprobará inmediatamente. En caso contrario, pasará a una segunda etapa de análisis (artículo 21).

- Eficiencias: ya en la segunda etapa, si la autoridad determina que la operación puede causar una restricción significativa de la competencia, podrá autorizarla si las partes logran probar que esta generará también ganancias de eficiencia que compensen tales efectos restrictivos (artículo 7).

Estos elementos funcionan como filtros para impedir que aquellas operaciones de concentración que probablemente no vayan a generar un impacto negativo significativo para la competencia no sean siquiera analizadas, ya sea por el tamaño de las empresas (umbrales) y la evidencia disponible (vía rápida), ni prohibidas (compensación por eficiencias), si bien no se define con claridad un estándar de prueba ${ }^{2}$. Esto responde a la idea, generalmente aceptada, de que el Derecho de la Competencia tiene por función administrar y reducir costos de error (falsos positivos y falsos negativos) y costos de administración, buscando la consecución de sus objetivos al menor costo posible (Easterbrook, 1984).

2 Sobre reglas y estándares en el análisis de operaciones de concentración, véase Ibáñez Colomo (2020). 
Los costos de error, no obstante, no pueden eliminarse. A pesar de no superar estos umbrales objetivos fijados por el DU 13-2019, es posible que empresas con significativas cuotas de mercado se concentren. Asimismo, si bien las limitaciones de la información disponible no permiten apreciar en un determinado momento los posibles efectos negativos de una operación aprobada por la vía rápida, es posible que estos se materialicen con el paso del tiempo.

Estas operaciones remanentes con efectos anticompetitivos no podrían ser prevenidas por el DU 13-2019 ni corregidas por la LRCA (al menos como esta suele ser entendida). Ante esto, procederemos a analizar una de las herramientas con las que cuentan otras autoridades de competencia para afrontar este problema: el control ex post de concentraciones.

\section{El control ex post de operaciones de concentración}

En otras jurisdicciones, a diferencia del Perú, las autoridades de competencia reconocen un sistema de control ex post de operaciones de concentración, a fin de complementar el régimen de control ex ante o sustituirlo, en caso de que no cuenten con uno.

\subsection{Experiencia comparada}

Entre las experiencias en otras jurisdicciones que podemos encontrar, tenemos las siguientes:

\section{- Reino Unido}

En el Reino Unido no existe un régimen de control ex ante de concentraciones obligatorio, por lo que su autoridad de competencia (Competition and Markets Authority - CMA) se ha visto en la necesidad de utilizar el control de conductas para implementar un control ex post de concentraciones.

A fin de ordenar este tipo de control, se ha establecido un régimen voluntario de control ex ante para concentraciones de gran magnitud. Asimismo, y con el objetivo de reducir la incertidumbre jurídica, la CMA solo puede realizar la primera fase del control ex post en un plazo de cuatro meses, el que inicia desde el momento en el que la autoridad haya podido tener conocimiento de la operación.

A través de este control ex post, la CMA tiene la facultad de imponer medidas correctivas estructurales o de actuar imponiendo medidas preventivas, 
lo cual puede permitir que se evite la culminación del acto de concentración en tanto se llevan a cabo las investigaciones por parte de la autoridad.

\section{- Bélgica}

A diferencia del Reino Unido, Bélgica es un país que cuenta con un régimen de control ex ante de concentraciones de obligatorio cumplimiento. Sin embargo, la autoridad de competencia belga (Belgian Competition Authority - BCA) también cuenta con facultades para ejercer un control ex post sobre aquellas operaciones que no se encuentran sujetas al control ex ante, utilizando para esto las facultades que le brinda la ley para realizar el control de conductas.

Es preciso señalar que, en el caso belga, estas facultades se basan en normas que tipifican el abuso de posición de dominio, las cuales se encuentran en el artículo IV.2 del Código Belga de Derecho Económico, en concordancia con la experiencia europea que surge del caso C-6/72 (Europemballage Corporation y Continental Can Company Inc contra la Comisión).

Como señala Johnckheere (2018), este ejercicio de autoridad por parte de la $\mathrm{BCA}$ no ha significado que el régimen belga haya caído en la incertidumbre, en tanto que se ha buscado darle un marco que permita un ejercicio estable y predecible del control de concentraciones.

Así, en primer lugar, se excluye de este control ex post a aquellas operaciones que han tenido que atravesar un control ex ante; en el caso de las operaciones que sí se encuentran sujetas a control ex post, se estableció el criterio de que todas las concentraciones se presumen legítimas, salvo la BCA demuestre lo contrario y que el análisis a aplicar debe ser el mismo que se utiliza en el control ex ante de concentraciones.

Por último, jurisprudencialmente se determinó que la BCA tiene la facultad de imponer medidas preventivas en aquellos escenarios en los que prima facie se evidencia una posible restricción de la competencia.

\section{- Luxemburgo}

De forma similar a lo que sucede en el Reino Unido, en Luxemburgo no se tiene un régimen de control de concentraciones explícito, sino que se cuenta con normativa que prevé el control de conductas. Sin embargo, a través de la 
Decisión 2016-FO-04, el Consejo de Competencia (autoridad de competencia de Luxemburgo) estableció un régimen de control ex post de concentraciones desde la perspectiva del abuso de posición de dominio, de forma similar al caso belga, sin negar la posibilidad de que se aplique el mismo criterio en caso de acuerdos (Gloden \& Veranneman, 2017).

A diferencia de Bélgica y Reino Unido, en Luxemburgo no se prevé que la autoridad pueda tomar medidas preventivas, tratándose de un control que debe realizarse de forma exclusiva luego de la operación de concentración.

\section{- Chile}

En Chile, hasta el 1 de junio 2017, no existió un régimen obligatorio de control ex ante obligatorio, por lo que las autoridades utilizaron el control voluntario, pudiendo revisar con posterioridad a su realización aquellas operaciones de concentración que representen riesgos para la competencia.

Sin embargo, y a diferencia de Reino Unido, nunca se terminó por implementar verdaderamente un control ex post en tanto que se prefirió reforzar un sistema de control ex ante voluntario, pues se consideraba que este era preferible al ex post, ya que este último generaba gran incertidumbre jurídica (González \& Huneeus, 2015).

Así, la falta de certidumbre jurídica caracterizó al modelo chileno, pues si bien no era posible someter a autorización previa una concentración, las autoridades chilenas todavía podían imponer medidas ante una concentración consumada, sin llegar a deshacer la operación. Ante esto, la OCDE (2014) señaló en una investigación que el régimen chileno carece de transparencia, seguridad jurídica y predictibilidad. Es por este motivo que, en dicho país, a partir de mediados de 2017 se implementó un control ex ante de concentraciones de carácter obligatorio.

Ahora bien, de acuerdo con el Decreto Ley 211, ley de competencia chilena, existe la posibilidad de que la Fiscalía Nacional Económica intervenga luego de consumada una operación de concentración, cuando esta no supere los umbrales, hasta dentro del plazo de un año. En recientes declaraciones, el fiscal nacional económico de dicho país, Ricardo Riesco, indicó que: "Estamos pensando en revisar si corresponde adecuar los umbrales actualmente, pero también con claridad que no lo estamos haciendo a raíz de las 'Killer Acquisitions', porque 
para eso tenemos la herramienta del artículo 48 inciso $9^{\circ}$ del DL 211. Chile tiene una verdadera joya de legislación, en el sentido de que sin perjuicio de que existan umbrales, tiene un año para perseguir aquellas operaciones de concentración que no han ingresado al sistema, porque no cumplian los umbrales" (Chung, 2020, párr. 16; el énfasis es nuestro).

Dicho artículo establece que "cuando las operaciones de concentración a que se refiere el inciso anterior no le sean notificadas voluntariamente al Fiscal Nacional Económico, éste podrá, dentro del plazo de un año, contado desde el perfeccionamiento de la operación, instruir las investigaciones que estime procedentes de conformidad con la letra a) del artículo 39”, el cual está referido a las investigaciones por infracciones a dicha ley. En ese sentido, si las operaciones de concentración califican como una infracción bajo los alcances del Decreto Legislativo 211, podrían ser intervenidas de oficio hasta dentro del plazo de un año.

\section{- Estados Unidos}

Estados Unidos posee un sistema mixto, en donde existe un régimen de control ex ante obligatorio para aquellas operaciones que se encuentren bajo los alcances de la Hart-Scott-Rodino Act, mientras que las operaciones que se encuentren excluidas de los alcances de dicha norma se encuentran sujetas al control de conductas, al poder configurar acuerdos monopolizadores o que restrinjan el comercio. En el caso de Estados Unidos, la Sherman Act distingue entre la monopolización y el intento de monopolización como ofensas o infracciones distintas, requiriendo solo la primera como precondición la existencia de posición de dominio (Hovenkamp, 2008).

Esta situación ha generado un ecosistema complejo, en el que conviven investigaciones que se pueden dar tanto a nivel federal como estatal, producto de las características del sistema estadounidense, ante lo cual han surgido propuestas como la SMARTER Act que buscan armonizar las funciones de las distintas autoridades como la FTC o el Departamento de Justicia (Dunlop \& Speiser, 2020). 


\section{- Francia}

Si bien Francia es un país que cuenta únicamente con un sistema de control ex ante obligatorio, viene evaluando con gran interés la implementación de un control ex post que complemente su sistema. Para ello, la autoridad de competencia francesa ha solicitado la opinión de, entre otras instituciones, la American Bar Association (2018) a través de un reporte.

Dicho reporte se restringió a analizar la viabilidad del control ex post para aquellas operaciones que no se encuentran sujetas a control ex ante, reconociéndole al primero un rol residual que puede servir para ayudar a que la autoridad de competencia pueda implementar acciones en transacciones que no han sido evaluadas pero que representan un gran riesgo para la competencia.

Asimismo, el reporte recomendó que, de implementarse el control ex post, este solo pueda ejercerse dentro de un plazo de un año de realizada la operación de concentración, en tanto que mientras mayor sea el plazo, será más difícil implementar medidas correctivas, a lo que se suma un gran incremento en la inseguridad jurídica.

De igual forma, se recomendó que se permita que las empresas que vayan a realizar operaciones que puedan estar sujetas a control ex post, puedan realizar consultas voluntarias a la autoridad, a fin de encontrar cierta seguridad en la realización de la transacción.

Partiendo de estos antecedentes que se encuentran en diversas jurisdicciones, se observa que, si bien se reconoce la utilidad que puede tener un sistema de control ex post de operaciones de concentración, se hace necesario que este tenga ciertos límites para un funcionamiento óptimo. En caso contrario, se generaría un entorno de inseguridad o incertidumbre jurídica para los agentes económicos.

Asimismo, se evidencia que en aquellos casos en los que coexisten el control ex post junto a un control ex ante obligatorio, resulta necesario tener disposiciones que eviten dudas sobre en qué casos cada uno resulta aplicable, recomendándose que el control ex post tenga un papel subsidiario con el fin de analizar aquellas concentraciones que hayan escapado de los umbrales o excepciones del control ex ante. 


\subsection{Ventajas y desventajas}

Frente a tantas jurisdicciones que de diversas formas vienen implementando un control ex post de concentraciones, resulta necesario que nos preguntemos sobre la utilidad de este tipo de control y si es que realmente sirve a los fines del Derecho de la Competencia. Esta pregunta es de relevancia en tanto que se puede alegar que cualquier posible utilidad del control ex post de concentraciones podría tornarse en irrelevante con la implementación de un control ex ante obligatorio.

A esto se puede responder, en palabras de Bullard (2019), señalando que "todos quisiéramos poder adivinar el futuro. Pero querer no es poder” (párr. 1). Y es que el control ex ante es, en esencia, la búsqueda de predecir cómo es que se desenvolverá un sistema caótico como lo es cualquier mercado como consecuencia de una operación de concentración.

Una característica de los sistemas caóticos es que cualquier modificación en las condiciones iniciales puede llevar a resultados abismalmente diferentes, bajo el llamado efecto mariposa (Math Vault, 2015). Esto lleva a que "any small extensión of the range of prediction requires an exponential improvement in the accuracy of the data" (Kaczynski, 2020, p. 14); en otras palabras, para poder producir una predicción en un sistema caótico resulta necesario contar con gran cantidad de datos e información precisos.

¿Cómo puede entonces una autoridad de competencia realizar un adecuado control ex ante? La autoridad se encuentra con grandes dificultades al momento de realizar este tipo de control, siendo el primero de estos el contar con la información suficiente para arribar a una decisión adecuada e imponer las medidas correspondientes de ser el caso.

Pese al desarrollo que pueda adquirir una autoridad de competencia con respecto a conseguir fuentes confiables y objetivas de información, a través de la mejora de la inteligencia económica de la organización o contratando para este fin a terceros especializados, la información brindada por las empresas participantes de la operación de concentración sigue siendo el principal insumo (Gotlieb \& Van Gerven, 2015). Esto coloca a la autoridad en una situación en la que su principal fuente de información es parcializada.

Teniendo en consideración esta dependencia de información proveniente de las empresas que buscan la concentración, se puede tomar en consideración la investigación realizada por Dertwinkel-Kalt y Wey (2016) a fin de encontrar los incentivos de estas empresas a brindar dicha información. Dichos autores encontraron que en aquellos 
sistemas en los que, como el europeo, se fomentaba la propuesta de condiciones por parte de las empresas, similar al que establece el DU 13-2019, se termina por generar un contexto que desincentiva la entrega de más información y por ende se termina imponiendo medidas débiles. Esta falta de información desaparece en gran medida en un control ex post, en tanto que en este la autoridad tendrá información real de cómo la concentración ha impactado en la competencia, lo que permitiría la imposición de medidas adecuadas.

De forma adicional a esto, como ya habíamos señalado, otra de las ventajas que tiene la aplicación de un control ex post es que sirve para reducir los costos de error que genera el control ex ante. Esto se debe a que la aplicación de este último suele limitarse a aquellas operaciones que superen determinados umbrales, a pesar de que esto pase por alto operaciones que podrían involucrar a empresas con participaciones de mercado significativas. De este modo, con un sistema que se base únicamente en el control ex ante se tendría un punto ciego en el control de concentraciones. Es por este motivo que Tropeano (2020), luego de realizar un análisis a fin de identificar el sistema que genere un mayor beneficio al consumidor (consumer surplus), encontró que este se daba en aquellos casos en los que el control de concentraciones se veía en un sistema que cuente con un control ex ante complementado por un control ex post.

Como se observa, nuevamente el control ex post muestra características que lo hacen ver, en abstracto, como un mecanismo más eficaz que el control ex ante. Sin embargo, como contrapartida se tiene la inseguridad jurídica que el control ex post supondría para las operaciones de concentración, en tanto que estas se encontrarían frente a una suerte de espada de Damocles con el miedo de que súbitamente la autoridad les inicie una investigación luego de consumada la concentración. Esto tendría como efecto desincentivar o volver onerosas las operaciones de concentración en general por la inseguridad de que, una vez consumado el acuerdo, la autoridad de competencia pueda deshacer el mismo e imponer cuantiosas multas a las empresas que participaron de la concentración.

Así, producto de la ponderación entre efectividad y seguridad jurídica, la experiencia comparada muestra cómo muchos países han establecido un control ex ante para aquellas operaciones que superen un determinado umbral, designándole al control ex post un rol residual, y limitado temporalmente. Sobre todo, en aquellos casos en los que la evidencia empírica acredite que la concentración no ha generado las eficiencias esperadas y ha tenido un efecto negativo en la competencia,

Es por este motivo que, incluso en aquellas jurisdicciones en las que se cuente con un control ex ante, el control ex post aparece como la herramienta subsidiaria que le 
permitirá a la autoridad de competencia perseguir aquellas operaciones de concentración que terminaron por tener un efecto nocivo en la competencia. De ahí que el control ex post se muestra como útil para alcanzar los fines del Derecho de la Competencia.

Finalmente, su éxito dependerá, como en toda política pública, de un diseño institucional y legal adecuado.

\section{4. ¿Es posible aplicar un control ex post de operaciones de concentración en el Perú?}

Ahora, si las operaciones de concentración, al igual que cualquier conducta, pueden tener efectos negativos y el control ex post es una política útil para afrontar este problema, no sería disfuncional al sistema peruano una interpretación de la LRCA o del DU 13-2019 que permita que el Indecopi revise, sancione o corrija estas acciones. Partiendo de esa premisa, y considerando que el DU 13-2019 se limita a las operaciones que superan los umbrales, analizaremos los alcances de la LRCA.

Esta ley tipifica como infracciones tres tipos de conductas anticompetitivas: abuso de posición de dominio (artículo 10), prácticas colusorias horizontales (artículo 11) y prácticas colusorias verticales (artículo 12), así como establece las consecuencias por tales inconductas: sanciones y medidas correctivas. En ningún caso puede establecerse una sanción o medida correctiva si no se ha determinado previamente, a través de un procedimiento administrativo, la existencia de una infracción.

\subsection{Abuso de posición de dominante}

En primer lugar, cabe preguntarse si las operaciones de concentración podrían calificar como un abuso de posición de dominio en el Perú, tal como sucede en otras jurisdicciones. Sin embargo, para arribar a esta conclusión hay que superar algunas dificultades que vienen desde la propia LRCA.

En primer lugar, la existencia previa de posición de dominio, en el sentido de que solo podría ser una operación de concentración en la que participe una empresa que ya es dominante la que pueda ser revisada de oficio a través de un control ex post por el Indecopi.

En segundo lugar, de la redacción del artículo 10.1 de la LRCA pareciera desprenderse la necesidad de una relación causal entre la posición de dominio y la conducta anticompetitiva, en el sentido de que, en ausencia de aquella, no sería posible 
realizar esta ${ }^{3}$. Así, por ejemplo, los actos de competencia desleal como la denigración o el engaño pueden ser revisados también en sede de libre competencia en jurisdicciones como España ${ }^{4}$ C Chile ${ }^{5}$, además de los ya mencionados supuestos de Estados Unidos o la Unión Europea para los casos de operaciones de concentración, a pesar de que en estos supuestos no es necesaria en abstracto la existencia de posición de dominio para llevar a cabo esas conductas.

Sin embargo, a propósito de la litigación predatoria, que es una modalidad de abuso de posición de dominio reconocida expresamente en la LRCA (art. 10.2, literal f), se ha señalado, con base en una interpretación de los antecedentes normativos de la LRCA y los objetivos de esta, que

...las conductas infractoras... no son tales porque únicamente un dominante puede realizarlas, sino porque la posición de dominio permite que un agente económico, al emprender tales conductas, pueda tener la capacidad de afectar de manera significativa las condiciones de competencia en el mercado, en beneficio propio y desmedro de los competidores. (2015)

Y es que, en efecto,

...no tendría sustento - $\mathrm{y}$ de hecho no lo tiene- señalar que un supuesto de abuso de posición de dominio deja de ser reprochable cuando la conducta en sí también puede ser realizada por agentes sin tal posición dominante. De ser

3 Decreto Legislativo 1034 - Ley de Represión de Conductas Anticompetitivas

Artículo 10.- El abuso de la posición de dominio.- 10.1. Se considera que existe abuso cuando un agente económico que ostenta posición dominante en el mercado relevante utiliza esta posición para restringir de manera indebida la competencia, obteniendo beneficios y perjudicando a competidores reales o potenciales, directos o indirectos, que no hubiera sido posible de no ostentar dicha posición (el énfasis es nuestro).

$4 \quad$ Ley 15/2007, de 3 de julio, de Defensa de la Competencia.

Artículo 3. Falseamiento de la libre competencia por actos desleales.

La Comisión Nacional de la Competencia o los órganos competentes de las Comunidades Autónomas conocerán en los términos que la presente Ley establece para las conductas prohibidas, de los actos de competencia desleal que por falsear la libre competencia afecten al interés público.

5 Decreto Ley $N^{\circ} 211$ que fija normas para la defensa de la libre competencia

Artículo $3^{\circ}$.- El que ejecute o celebre, individual o colectivamente cualquier hecho, acto o convención que impida, restrinja o entorpezca la libre competencia, o que tienda a producir dichos efectos, será sancionado con las medidas señaladas en el artículo 26 de la presente ley, sin perjuicio de las medidas preventivas, correctivas o prohibitivas que respecto de dichos hechos, actos o convenciones puedan disponerse en cada caso.

Se considerarán, entre otros, como hechos, actos o convenciones que impiden, restringen o entorpecen la libre competencia o que tienden a producir dichos efectos, los siguientes:

c) Las prácticas predatorias, o de competencia desleal, realizadas con el objeto de alcanzar, mantener o incrementar una posición dominante. 
así, no podrían ser sancionadas ninguna de las conductas recogidas en la Ley de Libre Competencia: la negativa de trato, la discriminación exclusoria, las ataduras o cualquier otra forma de boicot. Porque para realizar dichas conductas anticompetitivas no se requiere de una posición de dominio. (Fernandez Flores, 2015)

En ese sentido, partiendo de los objetivos de la ley, considerando la experiencia de otras jurisdicciones y también que existen conductas reconocidas en la LRCA como el abuso de procesos legales que, en estricto, no requieren de la existencia de posición de dominio para considerarse como infracciones, es posible una interpretación de la LRCA en el sentido de que las operaciones de concentración cuando son realizadas por agentes económicos dominantes pueden calificar como infracciones por abuso de posición de dominio. Por supuesto, habría que acreditar que dicha operación ha sido realizada con el fin de perjudicar a competidores, que no apareja ninguna eficiencia socialmente relevante o que el impacto negativo en la competencia es de una magnitud tal que amerita una intervención, de conformidad con los artículos 9 y 10 de la LRCA.

Esta interpretación, no obstante, tiene una gran limitante, que radica principalmente en la ausencia de investigaciones y casos en la experiencia peruana contra operaciones de concentración, bajo el entendimiento de que no ha existido el control de estructuras en el Perú. No obstante, consideramos que el texto de la LRCA brinda suficiente margen como para que esta posición pueda discutirse ante las autoridades administrativas y judiciales, quienes definirán en un futuro esta posibilidad.

\subsection{Prácticas colusorias horizontales y verticales}

Posteriormente, corresponde analizar si de la redacción de los artículos 11 y 12 de la LRCA se puede considerar a las operaciones de concentración como prácticas colusorias. En nuestra consideración, son posibles dos interpretaciones válidas sobre el concepto de prácticas colusorias. En primer lugar, a partir de la literalidad, como un actuar coordinado entre agentes económicos, el cual puede celebrarse mediante acuerdos, prácticas concertadas (definidas como un acuerdo tácito o de menor intensidad), recomendaciones y decisiones (las cuáles, en estricto, no consisten en un actuar coordinado). En segundo lugar, a partir de sus modalidades, como un acuerdo, práctica concertada, recomendación o decisión, lo que implicaría que no necesariamente estaríamos frente a un actuar coordinado (caso que se reservaría solo para las prácticas concertadas), sino también ante actos instantáneos que pueden o no resultar en una cooperación práctica (acuerdos, recomendaciones y decisiones). 
La primera interpretación se construye a partir de la definición literal de sus términos. Así, se entiende por "práctica"6 al ejercicio o realización de una actividad de forma continuada y por "colusión"7 a aquel pacto ilícito en daño de terceros, lo que pone de relieve el criterio de pluralidad. Una práctica colusoria (horizontal o vertical) para los efectos de la LRCA, entonces, vendría a ser el pacto entre dos o más agentes económicos para realizar entre sí de forma continuada una actividad ilícita en daño de terceros. Por definición, estas se tratarían de infracciones continuadas ${ }^{8}$.

Dado que la infracción consiste en el desarrollo concertado de una actividad ilícita, la pluralidad de participantes es necesaria tanto al momento de celebrar el acuerdo, como es lógico, pero también al momento de ejecutarlo, pues de lo contrario no estaríamos frente a un actuar coordinado en el mercado al tratarse de un solo agente económico. Por ejemplo, una fusión entre dos competidores o la adquisición de la planta de producción competidora no calificarían como una práctica colusoria horizontal. Es cierto que al momento del pacto intervino más de un agente económico, pero ello no genera un actuar coordinado: la fusión consolida a los competidores en uno y la transferencia de propiedad de la planta no supone que las partes coordinen su comportamiento o estrategia competitiva (si bien podría cambiar).

A este actuar coordinado se llegaría a través de un "acuerdo" o de una "práctica concertada". La jurisprudencia y la doctrina han intentado dar múltiples explicaciones sobre ambos conceptos, pero actualmente se entienden como un mismo fenómeno con intensidades distintas. En el caso seguido por la Comisión Europea contra Anic Partecipazioni SpA, se precisó que ambos conceptos "comprenden formas de colusión que tienen la misma naturaleza y solo pueden distinguirse entre sí por su intensidad y por las formas en que estos se manifiestan en la realidad" (Tribunal de Justicia de la Unión Europea, 1999).

Los acuerdos, entonces, estarían referidos al concierto de voluntades expreso (formalizado o no) y las prácticas concertadas, más bien, a un entendimiento tácito, pero siendo lo relevante sustituir la independencia en el mercado por una cooperación práctica (Gorab \& Greene, 2019).

Siguiendo la jurisprudencia europea, la Comisión de Defensa de la Libre Competencia del Indecopi, resolvió señalando que el elemento esencial de toda práctica

\footnotetext{
6 De acuerdo con la novena acepción en el Diccionario de la Real Academia Española: "Uso continuado, costumbre o estilo de algo". También entendido como: "Ejercicio o realización de una actividad de forma continuada y conforme a sus reglas" (s.f.). Véase también Lexico.com (s.f.).

7 Según el Diccionario de la Real Academia Española: "Der. Pacto ilícito en daño de tercero" (s.f.).

8 Véase el Expediente 011- 2015/CLC de la Resolución 157-2019/SDC-INDECOPI del 26 de agosto de 2019 (Sala Especializada en Defensa de la Competencia del Tribunal del Indecopi, 2019).
} 
colusoria horizontal es una conducta coordinada que puede tener diversas formas de materialización, siendo que "el punto más importante para determinar la participación de un agente en este tipo de conductas es acreditar, en base a las herramientas descritas previamente, que el agente imputado modificó su actuar independiente por un comportamiento de colaboración". Para tales efectos, podrán valerse de todos los medios probatorios permitidos por ley para acreditar, directa o indirectamente, la práctica colusoria horizontal (Comisión de Defensa de la Libre Competencia del Indecopi, 2018).

Ahora bien, también se incluyen entre las prácticas colusorias a las decisiones y recomendaciones. Estas conductas, en estricto, no responden a la definición de actuar coordinado, pudiendo incluso ser muchas veces unilaterales. Es cierto que están relacionadas con un actuar coordinado en tanto que al promoverlo podrían dar pie a su surgimiento, pero de ello no puede deducirse que sean en sí mismas la sustitución del actuar independiente por uno coordinado. De hecho, no son "prácticas" (pues son instantáneas) ni "colusorias" (al menos no en todos los casos), pero pueden afectar a la competencia.

La Sala Especializada en Defensa de la Competencia del Tribunal del Indecopi señaló que estas conductas

\begin{abstract}
...consisten en declaraciones o indicaciones destinadas a uniformizar el comportamiento de sus miembros, restringiendo la competencia entre ellos o frente a terceros, con los efectos negativos que de ello se derivan... [En concreto,] constituyen decisiones si tienen carácter vinculante, en virtud de las normas de la asociación o gremio involucrado, o recomendaciones si tienen únicamente carácter orientativo, pero poseen la capacidad para influir en el comportamiento de los agentes económicos a las que van dirigidas (2019).
\end{abstract}

Estas declaraciones, por su propia naturaleza, no son una actuación coordinada en el mercado. Son declaraciones instantáneas que quedan consumadas con el único acto (colegiado o no) de decidir o de recomendar. Por ello, el actuar coordinado que estos actos puedan generar puede ser perseguido de manera independiente: "Ahora bien, la responsabilidad de una asociación o gremio por las decisiones o recomendaciones que realice no enerva que se pueda atribuir responsabilidad a sus asociados o agremiados en la medida de que, adicionalmente a tales decisiones o recomendaciones, los miembros de estas entidades hayan realizado un acuerdo anticompetitivo. En efecto, para evitar que algún agente eluda su responsabilidad, estas conductas pueden ser analizadas como decisiones o recomendaciones de la asociación o gremio y/o como acuerdos entre sus 
asociados o agremiados" (Sala Especializada en Defensa de la Competencia del Tribunal del Indecopi, 2017).

En esta línea se ha señalado que "llama notoriamente la atención que INDECOPI también haya investigado y sancionado a personas naturales, en su condición de directivos de asociaciones o gremios de empresas, por prácticas de recomendación, sin abrir procedimiento ni condenar por la misma infracción a las agrupaciones de empresas a las que pertenecían esos directivos". Por esa razón, "si sólo se acusa por recomendaciones a los directivos de los gremios o asociaciones de empresas, se pierde el 'elemento esencial consistente en la conducta coordinada' que debe encontrarse presente en toda práctica colusoria horizontal” (Quintana Sánchez, 2015).

En consecuencia, en la práctica han sido sancionados casos de prácticas colusorias que, como indicamos, no son "prácticas" (pues son instantáneas) ni "colusorias" (al menos no en todos los casos), pero que sí pueden afectar a la competencia.

En ese contexto, queda abierta la posibilidad de proponer otra interpretación de los artículos 11 y 12, ya no a partir de la literalidad de la frase "prácticas colusorias", sino a partir de sus disímiles y variadas modalidades y sus respectivos conceptos, que podría permitir una cobertura más amplia de conductas anticompetitivas, en pro de la eficiencia económica en los mercados. Ello teniendo en cuenta que también existen acuerdos que no necesariamente implican un actuar coordinado, pero, aun así, son anticompetitivos, como veremos más adelante.

Así, los artículos 11 y 12 prohibirían acuerdos, recomendaciones, decisiones (que no necesariamente implican un actuar coordinado ni requieren de pluralidad) y prácticas concertadas (que sí lo hace) cuando estas tengan por objeto u efecto restringir la competencia en el mercado, permitiendo a los infractores obtener beneficios por razones distintas a una mayor eficiencia económica. Probablemente, hubiera sido más conveniente denominar a este grupo como conductas anticompetitivas de efectos horizontales o verticales, según corresponda, pues eso es lo que tienen en común y no el hecho de ser una práctica.

Nótese que esta interpretación parte de que las recomendaciones y decisiones no son "prácticas", característica que comparten también con los acuerdos (no así la unilateralidad). De hecho, atendiendo a una definición básica de acuerdo como concierto de voluntades, es posible que también agentes económicos competidores celebren un acuerdo con un objeto o efecto anticompetitivo a pesar de que ello no implique coordinar su actuación en el mercado. Esa situación no se trataría de una infracción continuada, pues no se compone de un conjunto de actuaciones bajo el control de los infractores, sino de un solo acto acordado que elimina o restringe la competencia. En términos de 
Derecho Administrativo Sancionador, se trataría de infracciones instantáneas con efectos permanentes.

Estos acuerdos serían los denominados actos u operaciones de concentración, los cuales son realizados por dos o más agentes económicos para eliminar la competencia entre sí, antes que para coordinar su actuar en el mercado. Como vimos en la sección 1, los actos de concentración y las conductas anticompetitivas en general están en la aptitud de reducir los niveles de eficiencia en los mercados en perjuicio de los consumidores. Los actos u operaciones de concentración más comunes son los siguientes:

- Una fusión de dos o más agentes económicos. En este acuerdo, dos o más agentes económicos eliminan la competencia entre sí, ya sea porque se consolidan en una nueva persona jurídica o porque una es absorbida por la otra. En el caso de las operaciones horizontales, la tensión competitiva entre ambos agentes desaparece, al igual que en un supuesto de cártel. El efecto puede ser el mismo a pesar de que ambas no compartan el objeto de coordinar la actuación en el mercado.

- La adquisición por parte de uno o más agentes económicos de derechos que le permitan ejercer el control sobre otros. Para que este supuesto implique un acuerdo entre agentes competidores, los derechos deben ser adquiridos del agente controlante. En caso contrario, no serían propiamente competidores.

- La constitución por dos o más agentes económicos independientes entre sí de una empresa en común, joint venture o cualquier otra modalidad que implique la adquisición de control conjunto sobre uno o varios agentes económicos, de tal forma que dicho agente económico desempeñe de forma permanente las funciones de una entidad económica autónoma.

- La adquisición por un agente económico del control directo o indirecto, por cualquier medio, de activos productivos operativos de otro u otros agentes económicos. Piénsese en el caso de la adquisición de la planta de producción del competidor, lo que tiene como consecuencia que este deje de participar en el mercado. Nuevamente, la competencia entre ambas desaparece, lo que podría impactar negativamente en los niveles de concentración y eficiencia económica. 
Todos estos actos de concentración caen en la definición de acuerdo, pues al momento de celebrarse son dos o más agentes económicos. No obstante, ya que luego de su realización resulta en una unidad económica, no puede tratarse de un actuar o actividad coordinada en el mercado (o, literalmente, práctica colusoria). En ambos casos, sin embargo, el acuerdo puede tener un objeto o efecto anticompetitivo contrario a los fines de la LRCA.

En ese contexto, si las decisiones y recomendaciones pueden ser prohibidas a pesar de no ser "prácticas" ni "colusorias", los acuerdos que no sean una "práctica" pero tengan un objeto o efecto anticompetitivo también deberían estar sujetos al control ex post de conductas regulado por LRCA, tal y como se hace en otros países. Así, pues, es posible una interpretación de la LRCA en este sentido.

Las operaciones de concentración tanto horizontales y verticales, al igual que las recomendaciones y las decisiones, no son prácticas colusorias en su sentido literal, pero todas son conductas o acciones que pueden tener por objeto o efecto restringir la competencia y reducir la eficiencia en un mercado. Esta interpretación es coherente con la finalidad de la LRCA, como señalamos en la sección 2, y no deja de lado a las que serían, en estricto, "prácticas colusorias", pues los acuerdos también pueden tener por objeto coordinar la actuación en el mercado, al igual que, como es natural, las prácticas concertadas.

Quedará en manos de la jurisprudencia del Indecopi lograr una definición de "prácticas colusorias" que se construya sobre los elementos comunes que puedan existir entre los acuerdos, prácticas concertadas, recomendaciones y decisiones. Hasta ahora, no se han podido conciliar las diferencias entre dichas modalidades, por lo que el único elemento común es el objeto o efecto anticompetitivo que dichas conductas puedan tener o generar.

\subsection{Reformas legislativas}

E1 23 de octubre de 2020, el Congreso de la República aprobó el Proyecto de Ley que establece el Control Previo de Operaciones de Concentración Empresarial (en adelante, el Proyecto), el que sustituirá al DU 13-2019 en caso sea promulgado. Al respecto, el Proyecto introduce distintas modificaciones de gran relevancia al régimen de control de concentraciones en el Perú. Sin embargo, para efectos del presente artículo, el más relevante se encuentra en el numeral 6.4.:

La Secretaría Técnica de la Comisión de Defensa de la Libre Competencia del Indecopi podrá actuar de oficio en los casos que haya indicios razonables para 
considerar que la operación de concentración puede generar posición de dominio o afecte la competencia efectiva en el mercado relevante.

La notificación de la operación será voluntaria para las partes cuando las empresas involucradas no alcancen los umbrales establecidos en el numeral 6.1.

La aplicación del presente numeral será regulada a través del Reglamento.

Se ha interpretado que este numeral amplía los alcances del control de concentraciones en el Perú, que en el DU 13-2019 se encontraba limitado a un régimen de notificaciones obligatorias únicamente para aquellas operaciones que superaban los umbrales de la norma, en tanto que permitiría que la autoridad investigue una operación que no ha superado los umbrales, las cuales estarían sujetas a un régimen de notificaciones voluntarias.

Sin embargo, el numeral 6.4. es particularmente escueto en tanto que lo único que dispone es que la autoridad se encuentra autorizada a "actuar de oficio" ante aquellas operaciones que afecten la competencia (superen o no el umbral), sin especificar qué acciones podría tomar. Por este motivo es que, en una primera lectura, las facultades otorgadas a la autoridad resultan vacías al no haberse determinado un régimen sancionador ni medidas que podrían imponerse a estas operaciones que, pese a no superar el umbral, terminan afectando la competencia.

Si bien el Proyecto señala que esta actuación de oficio será regulada por el Reglamento, este no podría crear sanciones o medidas correctivas en contra de las operaciones que no hayan superado los umbrales, dado que este tipo de operaciones no se encuentra sujeta a un régimen de notificaciones obligatorias por lo que no se les podría imponer una sanción o medida correctiva por no notificar ni mucho menos condicionar la operación de concentración a una autorización por parte del Indecopi. Ello entraría en abierta contradicción con el alcance del régimen obligatorio de notificaciones.

De este modo, este "actuar de oficio" que introduce el Proyecto no tendría utilidad práctica, pues no contaría con un elemento disuasorio dentro del texto de la norma. En esa línea, los agentes económicos no tendrían incentivos para notificar de manera voluntaria al no estar expuestos a ningún tipo de riesgo. Sin embargo, esta primera lectura resultaría errónea si se tiene en cuenta lo desarrollado en los párrafos anteriores, sobre los alcances de la LRCA, que permitirían la existencia del control ex post de concentraciones a través de procedimientos administrativos sancionadores. 
Partiendo de esta interpretación, el "actuar de oficio" de la autoridad ante aquellas operaciones que, pese a no superar los umbrales, ocasionan daños anticompetitivos, debe enmarcarse dentro de las facultades que otorga la LRCA al Indecopi para combatir aquellas prácticas anticompetitivas. De este modo, una operación de concentración que no supera el umbral pero que genera una afectación a la competencia, si bien no podrá ser sancionada por la falta de notificación, puede ser evaluada como una práctica anticompetitiva al amparo de la LRCA. Como se sabe, esta última permite la imposición de multas y de medidas correctivas, por lo que se podría exigir incluso que se deshaga la operación de concentración.

Esta interpretación del numeral 6.4. también guarda coherencia con respecto a su segundo párrafo ya que, ante la posibilidad de que una operación de concentración pueda ser sancionada bajo la LRCA, se abre la posibilidad de que precautoriamente los interesados realicen una notificación voluntaria a fin de evitar intervenciones por parte de la autoridad una vez que se haya consumado la concentración.

En este sentido, la interpretación adecuada del numeral 6.4., a fin de que esta no sea una disposición carente de elementos disuasorios y que, por lo tanto, no pueda cumplir con su finalidad, es que constituye un reconocimiento del legislador de que una operación de concentración puede constituir una práctica anticompetitiva y por ende puede ser sancionada y/o corregida al amparo de la LRCA.

\section{Condiciones para un sistema de control ex post}

Entonces, ¿cómo se debería implementar el control ex post de concentraciones en el Perú? Partiendo de lo señalado en el anterior apartado, es posible sostener que el Indecopi cuenta con las facultades para realizar un control ex post de concentraciones, por lo que parecería no ser necesaria la dación de una nueva norma con rango de ley.

Sin embargo, y aprendiendo las lecciones de la experiencia chilena antes reseñada, en donde la incertidumbre terminó por minar los aspectos positivos que un control ex post pudiese tener, es necesario que exista una regulación adicional, ya sea a través de un Decreto Supremo que reglamente la LRCA o mediante lineamientos o directivas internas del Indecopi que limiten su discrecionalidad, si es que no también la discusión y aprobación de una nueva Ley que pueda conciliar los alcances de la LRCA, el DU 132019 y el control ex post con total certidumbre.

Al respecto, sobre lo desarrollado previamente, consideramos que las condiciones para un adecuado control ex post son las siguientes: 


\section{- Establecimiento del rol subsidiario del control ex post}

Teniendo en consideración que el DU 13-2019 establecerá por primera vez en el Perú un control ex ante de concentraciones general y de carácter obligatorio, a fin de resguardar la confianza legítima de las empresas que se vean sometidas a este control previo, el control ex post deberá ser aplicado únicamente a aquellas operaciones que no se encuentren sujetas a dicho Decreto de Urgencia.

Esto significa que este tipo de control se aplicará cuando no se llegue a los umbrales establecidos, así como a aquellos actos de concentración excluidos por el artículo 16.3. del Decreto de Urgencia (operaciones de concentración empresarial que incluyan empresas del sistema financiero que captan depósitos del público o empresas de seguros, que presentan riesgos relevantes e inminentes, que comprometan la solidez o estabilidad de las referidas empresas o de los sistemas que integran, las cuales serán evaluadas únicamente por la SBS).

En este último supuesto resulta aplicable el control ex post en tanto que ya habría sido superado el riesgo inminente que justifica la excepción. Sin embargo, esto no significa que el Indecopi pueda imponer medidas que puedan generar riesgos sistémicos en el sistema financiero.

\section{- Consultas con autoridades sectoriales}

A fin de evitar medidas que generen riesgo sistémico en el sistema financiero peruano, el Indecopi deberá solicitar informes tanto a la SMV como a la SBS en caso el control ex post se realice sobre empresas reguladas y supervisadas por estas.

De igual modo, y a fin de tener mayor conocimiento sobre los mercados y las mejores medidas a imponer, el Indecopi podrá solicitar a las autoridades sectoriales de aquellos mercados regulados la información que considere pertinente.

\section{- Límites temporales}

A fin de mitigar la inseguridad jurídica que puede generar la existencia de un control ex post, resulta necesario que se impongan límites a la facultad 
para investigar y sancionar. Al respecto un límite razonable es el de 1 año desde la realización de la operación de concentración y, en aquellos casos en los que se trate de diversas operaciones de concentración en el mismo mercado, que este plazo se cuente a partir de la última de estas. Naturalmente, al tratarse de infracciones (bajo la interpretación de la LRCA que estamos describiendo), esta modificación debería hacerse por Ley, pues dicha norma ya establece un plazo de prescripción de la potestad sancionadora de cinco (05) años.

Este plazo no solo responde a la mitigación de la inseguridad jurídica, sino también al hecho de que, conforme pasa el tiempo, cada vez resulta más difícil imponer medidas que reparen el daño a la competencia causado.

\section{- Permitir notificaciones voluntarias}

A fin de brindar un puerto seguro a aquellas operaciones que no se encuentran sujetas al control establecido por el DU 13-2019, se debe brindar la posibilidad de que las empresas involucradas puedan realizar una notificación voluntaria bajo el régimen establecido por el Decreto de Urgencia.

Si bien dicho control voluntario no podría por sí mismo detener la operación de concentración, sí podría servir para que las empresas involucradas tengan mayor seguridad de la transacción a realizar en caso de dudas, permitiendo la generación de un entorno de negociación con el Indecopi a fin de poder implementar medidas que disminuyan la afectación a la competencia que la operación pudiese ocasionar.

\section{- Fijación de estándares elevados}

Ante la amplitud que podría tener el uso del control ex post de concentraciones, resulta necesario que se fijen estándares que establezcan que esta herramienta será utilizada únicamente en escenarios en los que el daño a la competencia sea elevado o que no se hayan generado las eficiencias que uno pudiese esperar producto de una concentración. Esto a fin de evitar que efectos producto de cualquier concentración (como el aumento del IHH o la reducción de competidores) sirvan como excusa para iniciar un procedimiento. 


\section{- Cálculo de multa e imposición de medidas correctivas}

Debido a la gran diferencia que tendrían estos casos con los presentados en el tradicional control de conductas, resulta necesario que se establezca una metodología del cálculo de multas diferente para este tipo de casos.

Asimismo, considerando que, en principio, las operaciones de concentración son eficientes y legítimas, se debe tener un sistema que se enfoque más en la imposición de medidas correctivas que de las multas. En esa línea, se debe tener en cuenta que el deshacer la concentración debe ser una medida extrema y subsidiaria únicamente aplicable en aquellos casos en los que no hay otra solución posible y en los que el daño a la competencia sea notorio.

\section{- Limitar el actuar de oficio}

Finalmente, a fin de evitar la sobrecarga de la administración y reducir la inseguridad jurídica que el control ex post pueda generar, es recomendable que las investigaciones que se realicen sobre estas concentraciones solo puedan realizarse a partir de una denuncia de parte.

De este modo, en caso existan afectaciones a la competencia, asociaciones de consumidores, gremios empresariales y, en general, todo aquel que acredite un interés legítimo, pueda denunciar la operación cuando se haya visto o puedan verse afectados producto de la concentración.

Cabe resaltar que el fin principal de estas disposiciones debe ser el otorgar seguridad jurídica y establecer un marco a fin de controlar el actuar del Indecopi. Es por este motivo que resulta necesaria la emisión de nueva normativa en lugar de regular el uso de estas facultades únicamente a través de la jurisprudencia del Indecopi.

\section{Conclusiones}

Las herramientas con las que cuenta el Derecho de la Competencia para perseguir sus fines son diversas. Sin embargo, todas estas se encuentran enlazadas teleológicamente al buscar conseguir una mayor eficiencia en el mercado. Es en búsqueda de este fin que autoridades de competencia a nivel del mundo han recurrido no solo al control de conductas, sino también al control de estructuras a través del control ex ante de concentraciones. 
Empero, el control ex ante, si bien es una poderosa herramienta para garantizar que los mercados conserven una estructura que permita la competencia, presenta fallas y limitaciones, debido, entre otras causas, a que ninguna autoridad se encuentra en la capacidad de predecir el futuro.

En ese contexto, el control ex post surge como una herramienta que permite llenar estas carencias, a costa de sacrificar la seguridad jurídica de cualquier operación de concentración, motivo por el que su aplicación debe ser dada en un marco adecuado que permita realizar una ponderación adecuada entre la eficiencia del control ex post y la pérdida de seguridad jurídica que genera.

Ante esto, se observó que, si bien en el Perú es posible interpretar la LRCA en el sentido de que permite realizar un control ex post de operaciones de concentración (cuestión que deberá ser definida por la jurisprudencia), lo que permitiría complementar el control ex ante que entrará en vigencia por el DU 13-2019, es necesaria realizar algunas modificaciones normativas para una adecuada implementación de esta herramienta, en pro de la eficiencia económica en los mercados.

\section{Referencias}

American Bar Association. (2018). Comments of the American Bar Association's Sections of Antitrust Law and Intenational Law Regarding the French Competition Authority's Consultation on Modernizing and Simplifying the French Merger Control Law. Autorité de la concurrence. https://www. autoritedelaconcurrence.fr/sites/default/files/aba_expost_sept18.pdf

Bullard, A. (2019). La bola de cristal. Perú21. https://peru21.pe/opinion/bola-cristal473414-noticia

Comisión de Defensa de la Libre Competencia del Indecopi. (2018). Expediente 0052016/CLC. Resolución 104-2018/CLC-INDECOPI del 31 de diciembre de 2018. https://servicio.indecopi.gob.pe/buscadorResoluciones/ getDoc?docID=workspace://Spaces Store/12a0914a-dcb5-43fb-99faef13b08e895c

Chung, O. (2020). Los abusos empresariales, principalmente colusiones, causaron el estallido social. Tecito. https://tecito.com/los-abusos-empresarialesprincipalmente-colusiones-causaron-el-estallido-social/ 
Decreto Legislativo 1034. (2008). Decreto Legislativo que aprueba la Ley de Represión de Conductas Anticompetitivas. 24 de junio de 2008. D.O. No. 10273. https:// elperuano.pe/NormasElperuano/2008/06/25/217892-3.html

Decreto Ley 211. (1973). Fija normas para la Defensa de la Libre Competencia. 22 de diciembre de 1973. D.O. No. 28584.

Decreto de Urgencia 013 del 2019. Decreto de Urgencia que establece el control previo de operaciones de concentración empresarial. 18 de noviembre de 2019. D.O. No. 15159. Presidencia de la República. https://busquedas.elperuano.pe/ download/url/decreto-de-urgencia-que-establece-el-control-previo-de-operadecreto-de-urgencia-n-013-2019-1828320-1

Dertwinkel-Kalt, M. \& Wey, C. (2016). Evidence Production in Merger Control: The Role of Remedies. Discussion Paper N. 217. Düsseldorf Institute for Competition Economics. https://www.econstor.eu/bitstream/10419/130192/1/856580112. pdf

Dunlop, L. \& Speiser, S. (2020). Merger control in the United States: overview. Thomson Reuters Practical Law.

Easterbrook, F. (1984). The Limits ofAntitrust. Chicago Unbound. https://chicagounbound. uchicago.edu/cgi/viewcontent.cgi?article $=2152 \&$ context=journal_articles

Fernández Flores, D. (2015). Litigación predatoria: ¿anticompetitiva o desleal? Revista de la Competencia y la Propiedad Intelectual, (20), 11-56. https://revistas. indecopi.gob.pe/index.php/rcpi/article/view/17

Gloden, L. \& Veranneman, K. (2017). Merger Control 2017 - Luxembourg. En J. Bellis, y P. Elliot (Ed.). Merger Control (3. ${ }^{\mathrm{a}}$ ed.). Sweet \& Maxwell.

González, A. \& Huneeus, F. (2015). Hacia un Sistema de Control Preventivo de Fusiones para Chile. Trabajos de Investigación en Políticas Públicas Departamento de Economía U. de Chile. N. 19. http://econ.uchile.cl/uploads/ publicacion/44ba469a7897db85c00b6a1ba0394f2e7b98ce20.pdf

Gorab, D. \& Greene, E. (2019). Colusión: Interacción entre la Economía y el Derecho. En V. Facuse \& A. Montoya (Eds.), Desafíos de la Libre Competencia en Iberoamérica. Thomson Reuters.

Gotlieb, M. \& Van Gerven, G. (2015). Data Gathering and Analysis: The Anatomy of a Merger Investigation in Europe. Fordham International Law Journal, 39(1). https://ir.lawnet.fordham.edu/cgi/viewcontent.cgi?article=2403\&context=ilj

Hovenkamp, H. (2008). The Antitrust Enterprise. Principle and Execution. Harvard University Press. 
Ibáñez Colomo, P. (2020). The Shaping of EU Competition Law. First Paperback Edition. Cambridge University Press.

Jonckheere, P. (2018). Alken-Maes / AB InBev (Bosteels) - A 'Residual Jurisdiction' for the Belgian Competition Authority to Assess Non-notifiable Mergers? Journal of European Competition Law \& Practice, 9(7), 454 - 458. https:// academic.oup.com/jeclap/article-abstract/9/7/454/4844029

Kaczynski, T. (2020). Anti-Tech Revolution: Why and How (2. ${ }^{\mathrm{a}}$ ed.). Fitch \& Madison.

Kwoka, J. (2015). Mergers, Merger Control, and Remedies. A retrospective analysis of U.S. Policy. The MIT Press.

Lexico.com. (s.f.). Práctica. Lexico.com. https://www.lexico.com/es/definicion/practica

Ley 15/2007. (2007, 04 de julio). De Defensa de la Competencia. Jefatura del Estado. Boletín Oficial del Estado, (159). https://www.boe.es/buscar/act. php?id=BOE-A-2007-12946

Organisation for Economic Cooperation and Development. (2016). Agency DecisionMaking in Merger Cases. Background Note by the Secretariat. https://one. oecd.org/document/DAF/COMP/WP3(2016)6/en/pdf

Organisation for Economic Cooperation and Development. (2014). Evaluación del Régimen de Control de Concentraciones en Chile. Santiago de Chile. https:// www.oecd.org/daf/competition/Chile\%20merger\%20control_ESP_nov14. pdf

Ottaviani, M. \& Wickelgren, A. (2008). Policy Timing under Uncertainty: Ex Ante versus Ex Post Merger Control. https://pdfs.semanticscholar. org/c $28 \mathrm{a} / 3 \mathrm{c} 4483077369 \mathrm{fda} 4 \mathrm{db} 01$ adc 7 cad 556 f 4 ce 53 .pdf? $\mathrm{ga}=2.73489618 .1591377228 .1593577589-57180342.1593215659$

Pindyck, R. \& Rubinfeld, D. (2009). Microeconomía (7. ${ }^{\mathrm{a}}$ ed.). Pearson.

Proyecto de Ley 5913/2020-CR. Proyecto de Ley que efectiviza el control previo de operaciones de concentración empresarial. https://leyes.congreso.gob. pe/Documentos/2016_2021/Proyectos_de_Ley_y_de_Resoluciones_ Legislativas/PL05913-20200805.pdf

Quintana, E. (2011). El objetivo de la ley de competencia peruana y la interpretación de las conductas prohibidas. Revista de la Competencia y la Propiedad Intelectual, 7(13), 19 - 59. https://revistas.indecopi.gob.pe/index.php/rcpi/ article/view/97 
Quintana, E. (2015). Peligros en la tipificación de Recomendaciones como Práctica Anticompetitiva en la Ley peruana. Ius et Veritas, 24(51), 46 - 63. http:// revistas.pucp.edu.pe/index.php/iusetveritas/article/view/15651

Real Academia Española. (s.f.). Práctica. Diccionario de la Real Academia Española. https://dle.rae.es/pr\%C3\%A1ctico

Real Academia Española. (s.f.). Colusión. Diccionario de la Real Academia Española. https://dle.rae.es/colusi\%C3\%B3n?m=form

Sala Especializada en Defensa de la Competencia del Tribunal del Indecopi (2017) Expediente 004-2011/CLC. Resolución 508-2017/SDC-INDECOPI del 22 de agosto de 2017. https://servicio.indecopi.gob.pe/buscadorResoluciones/ getDoc?docID=workspace://SpacesStore/32efd791-ec4b-4d02-9293ea4813cfc68b

Sala Especializada en Defensa de la Competencia del Tribunal del Indecopi. (2019). Expediente 011-2015/CLC. Resolución 157-2019/SDC-INDECOPI del 26 de agosto de 2019. https://servicio.indecopi.gob.pe/buscadorResoluciones/ getDoc?docID=workspace://SpacesStore/0759ca43-e517-454d-93272b26ee0ff9f9

Tribunal de Justicia de la Unión Europea (1999) Asunto C-49/92 P. Sentencia del 8 de julio de 1999. https://curia.europa.eu/juris/showPdf.jsf?text=\&docid=44311\&pageIndex $=0 \&$ doclang $=$ es \&mode $=1$ st $\&$ dir $=\&$ occ $=$ first $\&$ part $=1 \&$ cid $=7110427$

Tropeano, J. (2020). Ex ante or Ex post? When the timing of merger assessment is up to the merger firms. École d)Économie de Paris - Paris School of Economics. https://www.parisschoolofeconomics.eu/docs/tropeano-jean-philippe/ endogetimingiep-rrfeb2020.pdf

Vault, M. (2015). The Definitive Glossary of Higher Mathematical Jargon. Math Vault. https://mathvault.ca/math-glossary/\#chaos

Viscusi, W.; Vernon, J. \& Harrington, Jr., Joseph E. (2010). Economics of Regulation and Antitrust (3. ${ }^{\mathrm{a}} \mathrm{ed}$.). The MIT Press. 

